\title{
Sexual Selection Mechanism for Agent-Based Evolutionary Computation
}

\author{
Rafał Dreżewski and Krzysztof Cetnarowicz \\ Department of Computer Science \\ AGH University of Science and Technology, Kraków, Poland \\ \{drezew, cetnar\}aagh.edu.pl
}

\begin{abstract}
Sexual selection mechanism can be used in evolutionary algorithms in order to introduce and maintain useful population diversity. In this paper the sexual selection mechanism for agent-based evolutionary algorithms is presented. Proposed co-evolutionary multi-agent system with sexual selection is applied to multi-modal optimization problems and compared to "classical" evolutionary algorithms.
\end{abstract}

Keywords: agent-based evolutionary computation, sexual selection mechanism.

\section{Introduction}

Evolutionary algorithms (EAs) are global optimization techniques based on principles of Darwinian model of evolutionary processes. Although they have been widely, and with great successes, applied to a wide variety of problems, EAs often suffer from loss of population diversity. This limits the adaptive capabilities of EAs, may lead to locating local optima instead of a global one, and limits the possibilities of application of EAs in some areas (multi-modal optimization and multi-objective optimization are only two examples). In the case of multi-modal optimization problems an EA without any special mechanisms will inevitably locate a single solution [7]. Multiple solutions can be found only after using some special multi-modal optimization techniques (so called niching techniques [7]). Niching techniques are aimed at forming and stably maintaining subpopulations (species) that are located within the basins of attraction of local optima of multi-modal problems.

The understanding of species formation processes (speciation) still remains the greatest challenge for evolutionary biology. The biological models of speciation include allopatric models (which require geographical separation of sub-populations) and sympatric models (where speciation takes place within one population without physical barriers) [5]. Sympatric speciation may be caused by different kinds of co-evolutionary interactions including sexual selection.

Sexual selection mechanism is the result of co-evolution of interacting sexes. Usually one of the sexes evolve to attract the second one to mating and the second one tries to keep the rate of reproduction — and costs associated with it—on optimal level (sexual conflict) [5]. The proportion of two sexes (females and males) in population is almost always $1: 1$. This fact combined with higher females' reproduction costs causes, that in the majority of cases, females choose males in the reproduction process according 
to some males' features. In fact, different variants of sexual conflict are possible. For example there can be higher females' reproduction costs, equal reproduction costs (no sexual conflict), equal number of females and males in population, higher number of males in population (when the costs of producing a female are higher than producing a male), higher number of females in population (when the costs of producing a male are higher than producing a female) [6].

Evolutionary multi-agent system (EMAS) is the agent-based realization of evolutionary computation. In such system three basic mechanism, which are responsible for initiating and maintaining evolutionary processes, exist: agents are able to reproduce, die, and there exist resources in the environment for which agents compete and which are needed for all their activities. The general model of co-evolution in multi-agent system (CoEMAS) [2] includes additionally the notions of species, sex and relations between species and sexes in evolutionary multi-agent system. These additional mechanisms can serve as a basis for creating techniques of maintaining useful population diversity and speciation in systems based on CoEMAS model. Computational systems based on CoEMAS model has already been applied with promising results to multi-modal optimization [3], and multi-objective optimization [4].

In the following sections the previous work on sexual selection as a population diversity maintaining and speciation mechanism for evolutionary algorithms is presented. Next, the co-evolutionary multi-agent system with sexual selection mechanism is presented. In such a system two sexes co-evolve: females and males. Female mate choice is based on values of some important features of selected individuals. Such system is applied to multi-modal function optimization and compared to "classical" niching techniques.

\section{Previous Research on Sexual Selection as a Speciation Mechanism}

Sexual selection is considered to be one of the ecological mechanisms responsible for sympatric speciation [5]. Gavrilets [5] presented a model, which exhibits three general dynamic regimes. In the first one there is endless co-evolutionary chase between the sexes where females evolve to decrease the mating rate and males evolve to increase it. In the second regime females' alleles split into two clusters both at the optimum distance from the males' alleles and males get trapped between the two female clusters with relatively low mating success. In the third regime males answer the diversification of females by splitting into two clusters that evolve toward the corresponding female clusters. As a result, the initial population splits into two species that are reproductively isolated.

Todd and Miller [10] showed that natural selection and sexual selection play complementary roles and both processes together are capable of generating evolutionary innovations and bio-diversity much more efficiently. Sexual selection allows species to create its own optima in fitness landscapes. This aspect of sexual selection can result in rapidly shifting adaptive niches what allows the population to explore different regions of phenotype space and to escape from local optima. The authors also presented the model of sympatric speciation via sexual selection. 
Sánchez-Velazco and Bullinaria [9] proposed gendered selection strategies for genetic algorithms. They introduced sexual selection mechanism, where males are selected on the basis of their fitness value and females on the basis of the so called indirect fitness. Female's indirect fitness is the weighted average of her fitness value, age, and the potential to produce fit offspring (when compared to her partner). For each gender different mutation rates were used. The authors applied their algorithm to Traveling Salesman Problem and function optimization.

Sexual selection as a mechanism for multi-modal function optimization was studied by Ratford, Tuson and Thompson [8]. In their technique sexual selection is based on the so called seduction function. This function gives a low measure when two individuals are very similar or dissimilar and high measure for individuals fairly similar. The Hamming distance in genotype space was used as a distance metric for two individuals. The authors applied their mechanism alone and in combination with crowding and spatial population model. Although in most cases their technique was successful in locating basins of attraction of multiple local optima in multi-modal domain, the strong tendency to lose all of them except one after several hundreds simulation steps was observed.

As it was presented here, sexual selection is the biological mechanism responsible for bio-diversity and sympatric speciation. However it was not widely used as maintaining population diversity, speciation and multi-modal function optimization mechanism for evolutionary algorithms. It seems that sexual selection should introduce open-ended evolution, improve adaptive capabilities of EA (especially in dynamic environments) and allow speciation (the formation of species located within the basins of attraction of different local optima of multi-modal fitness landscape) but this is still an open issue and the subject of ongoing research.

\section{Sexual Selection Mechanism for Co-evolutionary Multi-agent System}

The system based on CoEMAS model with sexual selection mechanism (SCoEMAS) can be seen in figure 1. The topography of the environment, in which individuals live, is graph with every node (place) connected with its four neighbors. There exist resource in the environment which is given to the individuals proportionally to their fitness function value. Every action (such as migration or reproduction) of individual costs some resource.

There are two sexes within the species living in the system: females and males. Reproduction takes place only when individuals have enough amount of resource. The genotypes of all individuals are real-valued vectors. Intermediate recombination and mutation with self-adaptation [1] are used for females and males.

The female's cost of reproduction is higher than male so their mating rate is lower. Each time step males search for the reproduction partners (females) in their neighborhood. Female chooses reproduction partner only if they are both located within the basin of attraction of the same local minima of multi-modal fitness landscape. Modified version of hill-valley function [11] is used in order to check if two individuals are located within the basin of attraction of the same local minima. Instead of three 


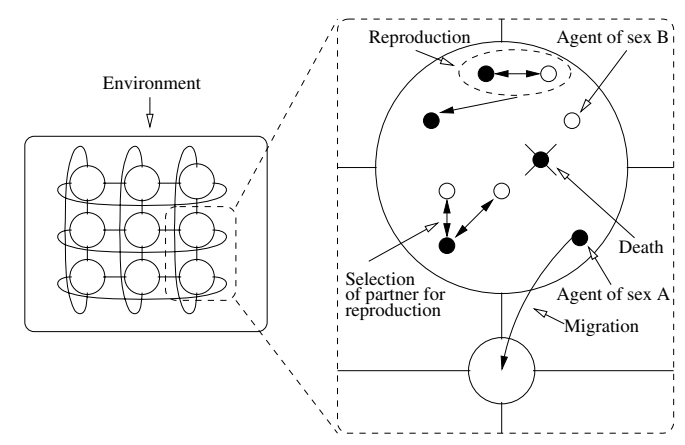

Fig. 1. Co-evolutionary multi-agent system with sexual selection mechanism

deterministically selected points, ten randomly generated points are used in order to evaluate hill-valley function value. The decision of acceptance is made on the basis of distance between female and male in phenotype space (Euclidean metric is used). The probability of acceptance is greater for more similar individuals. Also, the operator of grouping individuals into reproducing pairs is introduced. If female chooses male for reproduction they move together within the environment and reproduce during some simulation steps.

The system was applied to multi-modal function optimization and run against four commonly used test functions.

\subsection{Experimental Results}

The presented system with sexual selection mechanism was, among others, tested with the use of standard Rastrigin and Schwefel multi-modal problems (see fig. 2]1. In order to give a kind of reference point two other algorithms-standard EMAS and deterministic crowding (DC) [7]-was run against the same set of test functions.

Rastrigin function used in experiments is given by:

$$
f_{1}(x)=10 * n+\sum_{i=1}^{n}\left(x_{i}^{2}-10 * \cos \left(2 * \pi * x_{i}\right)\right) \quad x_{i} \in[-2.5 ; 2.5] \text { for } i=1, \ldots, n
$$

where $n$ is the number of dimensions ( $n=2$ in all experiments). The function has 25 local minima for $x_{1}, x_{2} \in[-2.5 ; 2.5]$.

Schwefel function is given by:

$$
f_{2}(x)=\sum_{i=1}^{n}\left(-x_{i} * \sin \left(\sqrt{\left|x_{i}\right|}\right)\right) \quad x_{i} \in[-500.0 ; 500.0] \text { for } i=1, \ldots, n
$$

\footnotetext{
${ }^{1}$ The presented CoEMAS was also tested with the use of other multi-modal problems, but because of space limitations it is out of scope of this paper.
} 


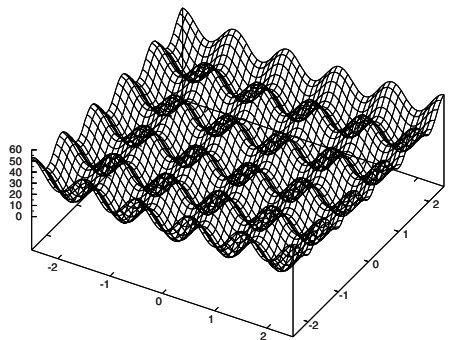

a)

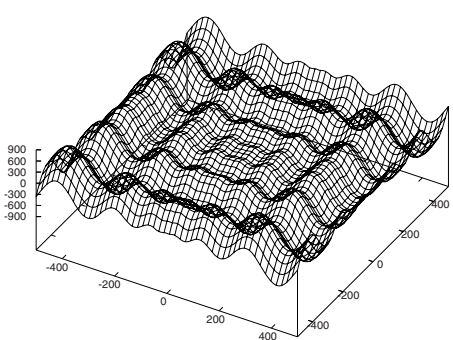

b)

Fig. 2. Rastrigin (a) and Schwefel (b) test functions

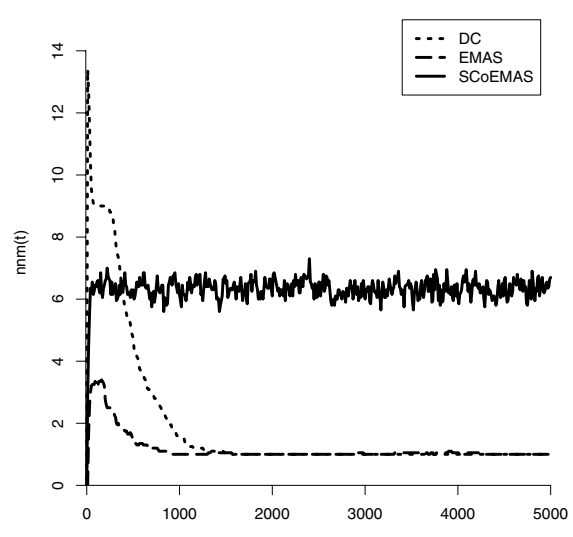

a)

Fig. 3. The number of located local minima neighborhoods of Rastrigin (a) and Schwefel (b) functions by CoEMAS with sexual selection, EMAS, and deterministic crowding technique (average values from 20 experiments)

This is deceptive function with unevenly distributed 62 local minima for $n=2$.

The figure 3 shows the average number of local minima neighborhoods located from 20 experiments. The local minima neighborhood was classified as located when there were at least three individuals closer than dist $_{\text {max }}=0.05$ from local minima for Rastrigin function and dist $_{\max }=10.0$ for Schwefel function. All the experiments were carried out for three techniques: SCoEMAS, EMAS, and DC.

The SCoEMAS stood relatively well when compared to other techniques. In all cases it formed and stably maintained species during the whole experiment. Although DC quickly located even greater number of local minima neighborhoods than other techniques, there was quite strong tendency to lose almost all of them during the rest part of experiment. Simple EMAS, without any niching mechanisms was not able to stably populate more than one local minima neighborhood. It turned out that in the case of multi-modal landscape it works just like simple EA. 


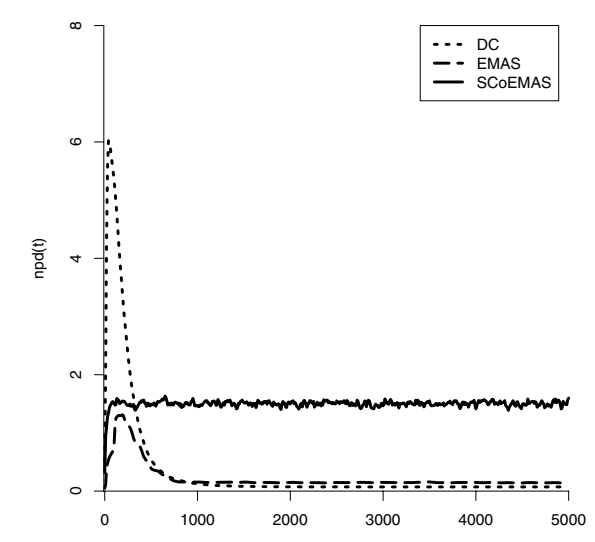

a)

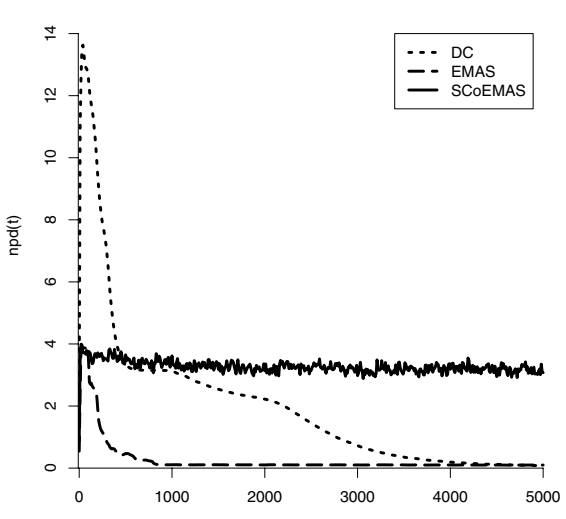

b)

Fig. 4. The value of proportional species' sizes indicator in experiments with Rastrigin (a) and Schwefel (b) functions (average values from 20 experiments)

Figure 4 shows the average values of proportional species' sizes indicator $n p d(t)$. The $n p d(t)$ indicator is defined as follows:

$$
\begin{gathered}
n p d(t)=\sum_{i=1}^{\left|D^{m i n}\right|} g\left(\left|A^{i}(t)\right|\right) \\
g\left(\left|A^{j}(t)\right|\right)= \begin{cases}1-\frac{|| A^{j}(t)\left|-n i_{o p t}^{j}\right|}{n i_{o p t}^{j}} & \text { if }\left|A^{j}(t)\right| \leq n i_{o p t}^{j} \\
1-\frac{|| A^{j}(t)\left|-n i_{o p t}^{j}\right|}{|A(t)|} & \text { if }\left|A^{j}(t)\right|>n i_{o p t}^{j}\end{cases} \\
n i_{o p t}^{j}=\frac{f^{\prime}\left(x_{j}^{+}\right)}{\sum_{k=1}^{\left|D^{m i n}\right|} f^{\prime}\left(x_{k}^{+}\right)}|A(t)|
\end{gathered}
$$

where: $D^{\min } \subseteq D$ is the set of local minima of the goal function $f, A(t)$ is the set of agents that exist in the system in time $t, x_{j}^{+}$is $j$-th local minima, $A^{j}(t)$ is the set of agents, that are closer than dist $_{\text {max }}$ to $j$-th local minima in the time $t, f^{\prime}=\delta \circ f$ is the modified goal function, $\delta: \mathbb{R} \rightarrow \mathbb{R}$ is scaling function which assures that the values of $f^{\prime}$ function are greater than 0 and that local maxima of this function are located in the same places as local minima of function $f$.

In the case when all sub-populations (species) located within the neighborhoods of local minima are of optimal sizes then $n p d(t)$ indicator has the maximal value (equal to the number of local minima). In the case when some subpopulations' sizes are not optimal then the value of this indicator falls down. The results presented in fig. 4 confirm that SCoEMAS stably maintains useful population diversity and that DC technique initially properly distributes individuals over the local minima basins of attraction, but then, as the time goes on, it loses almost all basins of attraction (species located within 


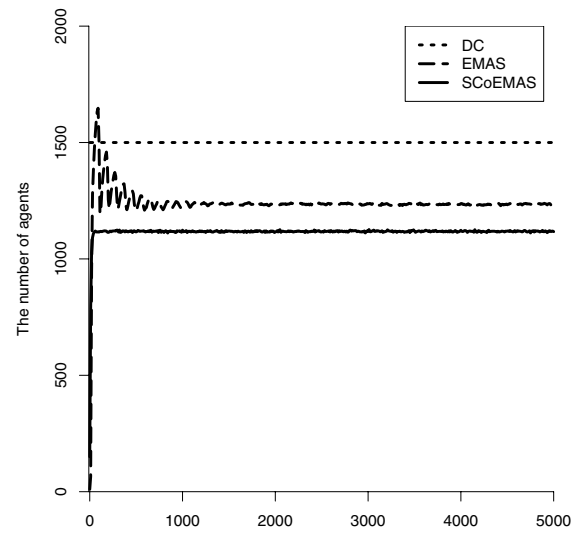

a)

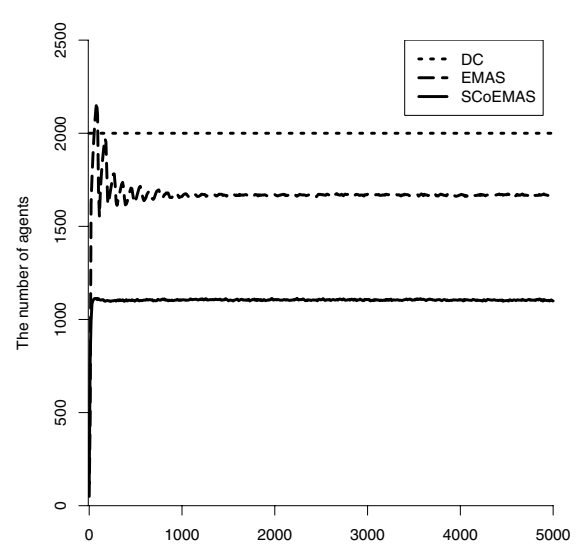

b)

Fig. 5. The number of individuals in population in experiments with Rastrigin (a) and Schwefel (b) functions (average values from 20 experiments)

them disappear). Also, earlier observations that EMAS is not able to maintain useful population diversity are fully acknowledged by the results presented in fig. 4

The sizes of population in EMAS, DC and SCoEMAS during experiments with Rastrigin and Schwefel functions are presented in fig. 5. EMAS and SCoEMAS used variable size populations, while DC population size was fixed (this results from the DC algorithm's assumptions [7]). In the case of EMAS and SCoEMAS systems initial population sizes are small and quickly adapts to the difficulty of the problem. It is worth noting that SCoEMAS uses the smallest population in experiments with both functions, what is the big advantage.

Presented results indicate that simple EMAS can not be applied to multi-modal function optimization without introducing special mechanisms such as co-evolution. DC technique has some limitations-it has the strong tendency to lose basins of attraction of "worse" local minima during the experiments (this fact was also previously observed in [12]). CoEMAS with sexual selection is able to form and stably maintain species but still more research is needed.

\section{Summary and Conclusions}

The general model of co-evolution in multi-agent system (CoEMAS) extends the basic EMAS model from single species and sex to multiple interacting species and sexes. On the basis of CoEMAS model computational and simulation systems may be developed. In this paper sample computational CoEMAS with sexual selection and resulting co-evolution of two sexes was presented. This system was applied to multi-modal function optimization. As presented results clearly show it properly formed and stably maintained species of agents located within the basins of attraction of local minima of multi-modal problems. SCoEMAS was able to detect and stably maintain more 
neighborhoods of local minima than EMAS without niching mechanism and deterministic crowding niching technique.

Future research will include the comparison of other variants of sexual conflict (different costs of reproduction for each sex, different costs of producing female and male individual, resulting in different proportions of individuals of each sex in population). Also, more detailed comparison to other "classical" niching and co-evolutionary techniques and the parallel implementation of systems based on CoEMAS model with the use of MPI are included in future research plans.

\section{References}

1. T. Bäck, D. Fogel, and Z. Michalewicz, editors. Handbook of Evolutionary Computation. IOP Publishing and Oxford University Press, 1997.

2. R. Dreżewski. A model of co-evolution in multi-agent system. In V. Mařík, et al., editor, Multi-Agent Systems and Applications III, volume 2691 of LNCS, pages 314-323, Berlin, Heidelberg, 2003. Springer-Verlag.

3. R. Dreżewski. Co-evolutionary multi-agent system with speciation and resource sharing mechanisms. Computing and Informatics, 25(4):305-331, 2006.

4. R. Dreżewski and L. Siwik. Multi-objective optimization using co-evolutionary multi-agent system with host-parasite mechanism. In V. N. Alexandrov, et al., editor, Computational Science - ICCS 2006, volume 3993 of Lecture Notes in Computer Science, pages 871-878, Berlin, Heidelberg, 2006. Springer-Verlag.

5. S. Gavrilets. Models of speciation: what have we learned in 40 years? Evolution, 57(10):2197-2215, 2003.

6. J. Krebs and N. Davies. An Introduction to Behavioural Ecology. Blackwell Science Ltd, 1993.

7. S. W. Mahfoud. Niching methods for genetic algorithms. PhD thesis, University of Illinois at Urbana-Champaign, Urbana, IL, USA, 1995.

8. M. Ratford, A. L. Tuson, and H. Thompson. An investigation of sexual selection as a mechanism for obtaining multiple distinct solutions. Technical Report 879, Department of Artificial Intelligence, University of Edinburgh, 1997.

9. J. Sánchez-Velazco and J. A. Bullinaria. Gendered selection strategies in genetic algorithms for optimization. In J. M. Rossiter and T. P. Martin, editors, Proceedings of the UK Workshop on Computational Intelligence (UKCI 2003), pages 217-223, Bristol, UK, 2003. University of Bristol.

10. P. M. Todd and G. F. Miller. Biodiversity through sexual selection. In C. G. Langton and T. Shimohara, editors, Artificial Life V: Proceedings of the Fifth International Workshop on the Synthesis and Simulation of Living Systems (Complex Adaptive Systems), pages 289-299. Bradford Books, 1997.

11. R. K. Ursem. Multinational evolutionary algorithms. In P. J. Angeline, Z. Michalewicz, M. Schoenauer, X. Yao, and A. Zalzala, editors, Proceedings of the 1999 Congress on Evolutionary Computation (CEC-1999), pages 1633-1640, Piscataway, NJ, USA, 1999. IEEE Press.

12. J.-P. Watson. A performance assessment of modern niching methods for parameter optimization problems. In W. Banzhaf, J. Daida, A. E. Eiben, M. H. Garzon, V. Honavar, M. Jakiela, and R. E. Smith, editors, GECCO-99: Proceedings of the Genetic and Evolutionary Computation Conference, volume 1, pages 702-709, San Francisco, CA, 1999. Morgan Kaufmann. 